A. Daglio, P. Malcovati and F. Maloberti

"A Multiplier-Free Digital RMS Calculation Unit for Integrated Microsystems"

Proceedings of International Workshop on Design of Mixed-Mode Integrated Circuits and Applications, Puerto Vallarta, Mexico, pp. 183-186, 1999 


\title{
A MULTIPLIER-FREE DIGITAL RMS CALCULATION UNIT FOR INTEGRATED MICROSYSTEMS
}

\author{
Alberto Daglio, Piero Malcovati and Franco Maloberti \\ Integrated Microsystems Laboratory, University of Pavia \\ Via Ferrata 1, 27100 Pavia \\ Tel. +390382 505205, Fax. +390382 505677 \\ E-Mail: alberto@ele.unipv.it, piero@ele.unipv.it, franco@ele.unipv.it
}

\begin{abstract}
This paper presents a novel 13-bit digital $R M S$ calculation unit, specifically designed to be integrated together with sensor and interface circuits in complete microsystems. The circuit exploits oversampling techniques (bitstream processing) to eliminate the area inefficient multipliers required in traditional $R M S$ calculation units. The proposed approach leads to a significant reduction (almost $30 \%$ ) in the silicon area required to implement the $R M S$ calculation algorithm for a 13 bit word.
\end{abstract}

\section{Introduction}

Modern electronic devices, including biomedical instruments, require accurate, reliable and non-invasive monitoring of physical or chemical signals. Integrated microsystems, therefore, are becoming very popular, because they combine reduced physical dimensions and low power consumption with high accuracy and low cost. Several integrated microsystems (e. g. current monitors or biological signal monitors), however, require the calculation of the root mean square $(R M S)$ value of the input signal. In this paper we propose an innovative digital $R M S$ calculation unit, specifically designed to be integrated together with sensor and interface circuits in a complete microsystem.

The block diagram of the complete microsystem is shown in Fig. 1. The signal to be measured is detected by a sensor, which produces a voltage output signal $V_{\text {sens }}$. Voltage $V_{\text {sens }}$ is then amplified, converted into the digital domain by a 13 bit ADC, processed in order to calculate the $R M S$ amplitude and finally delivered to an $\mathrm{I}^{2} \mathrm{~S}$ serial interface. The amplifier provides digitally programmable gain $(8$ bit) and offset ( 7 bit plus sign), in order to achieve better than $1 \%$ overall accuracy on the absolute value of the measurand. Two different operating ranges are available with independent calibration coefficients.

\section{RMS Calculation Methods}

The root-mean-square $(R M S)$ amplitude is an important property of time-dependent signals, because it is the only

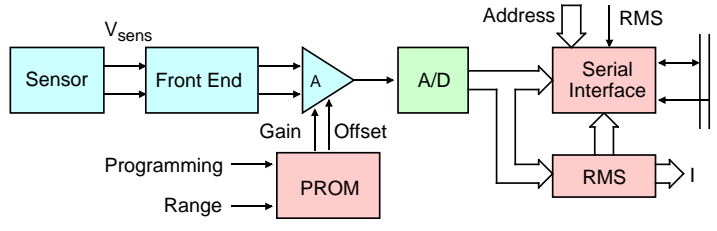

Fig. 1 -Block diagram of the complete microsystem

parameter independent of the waveform shape. The $R M S$ amplitude of an AC signal is defined as the DC signal which produces the same amount of heat in an ideal ohmic resistor [1]. The mathematical definition of the $R M S$ amplitude is

$$
x_{R M S}=\sqrt{\lim _{T_{A} \rightarrow \infty} \frac{1}{T_{A}} \int_{0}^{T_{A}} x^{2}(t) d t},
$$

where $x(t)$ denotes the time dependent signal and $T_{A}$ the averaging time. Moreover, the $R M S$ amplitude of a stochastic signal is equal to its standard deviation, while for periodic signals $T_{A}$ denotes the period. If uncorrelated signals (such as signals at different frequencies) are added, the $R M S$ amplitude of the sum corresponds to the square root of the sum of the squares of the individual $R M S$ amplitudes. The $R M S$ amplitude of a signal with DC $\left(x_{D C}\right), \mathrm{AC}\left(x_{A C}\right)$ and noise $\left(x_{N}\right)$ components is, therefore, given by

$$
x_{R M S}=\sqrt{x_{D C}^{2}+x_{A C}^{2}+x_{N}^{2}} .
$$

Two different methods are generally used to determine the $R M S$ amplitude of an AC signal, namely indirect $R M S$ detection and direct or true $R M S$ detection [2]. The indirect $R M S$ detection method is based on knowledge of the signal waveform. This method can be implemented, for example, with a full-wave rectifier, followed by a peakdetector. In this case, the $R M S$ amplitude $\left(x_{R M S}\right)$ is derived from the peak amplitude $(\hat{x})$ using the crest factor $C F$, defined as

$$
C F=\frac{\hat{x}}{x_{R M S}}
$$


Of course, the drawback of this $R M S$ detection method is its limitation to a defined waveform (for example a sinusoid or a square wave), which makes it unsuitable for accurate measurement of generic time-dependent signals. Moreover, it does not allow DC signals to be measured, thus leading to a large error in the presence of a DC component superimposed on the AC signal. Nevertheless, instruments based on indirect $R M S$ detection are widely used, because they are easy to design and cost-effective.

Direct or true RMS detection can be performed by computation or physical conversion. The computational approach, used in the proposed system, is based on the analog or digital implementation of Eqn. (1) with electronic building blocks. By contrast, the physical conversion method exploits non-linear transformations of the signal from the electrical domain to the mechanical or the thermal domain. For example, in an electrodynamometer the magnetic field of a fixed coil interacts with the magnetic field of a movable coil. Since the torque produced is proportional to the square of the current in the coils and given the system inertia, it is possible to obtain the $R M S$ amplitude of the current by measuring the displacement of the movable coil. Similarly, the electrostatic $R M S$ detector, exploits the quadratic electrostatic attraction between the movable plates of a capacitor, combined with their inertial dumping. Finally, the thermal $R M S$ converter uses the quadratic relationship between the voltage across a resistor and the corresponding dissipated power. The squared AC signal is averaged by the thermal inertia of the converter. By measuring the temperature difference produced by the dissipated power, it is, therefore, possible to determine the $R M S$ amplitude of the input signal.

\section{Oversampled RMS Calculation}

In integrated microsystems with an on-board A/D converter, the digital computational approach represents the most suitable, flexible and accurate solution for realizing the $R M S$ calculation unit. However, a direct implementation of Eqn. (1) in the digital domain with more than 8 bits of resolution, consumes a large amount of silicon area, because of the multipliers required. Yet, sensor signals typically have a small bandwidth, thus allowing the use of oversampling techniques (sigma-delta modulators) to reduce digital complexity, while maintaining reasonable speed of operation.

Oversampling techniques and, in particular, sigma-delta modulators, in fact, allow us to trade accuracy and hence complexity with speed of operation. Basically, by using a sigma-delta modulator an $N$-bit digital word (in the case considered $N=13$ ) can be reduced to a single-bit word sampled at higher frequency without any loss of information. The digital signal processing, then, can be performed on the single-bit word, thus reducing circuit complexity. The single-bit word obtained at the output of a sigma-delta modulator is usually called bitstream.
In a bitstream the information is coded in pulse density modulation (PDM) format. Assuming the input dynamic range of the sigma-delta modulator to be $\left[V_{L}, V_{H}\right]$, an output bit sequence containing $N_{1} 1$ 's and $N_{0} 0$ 's corresponds to an input signal

$$
V=V_{L}+\left(V_{H}-V_{L}\right) \frac{N_{1}}{N_{1}+N_{0}} .
$$

A sequence containing only zeros corresponds to the lower limit of the input range $\left(V_{L}\right)$, while a sequence containing only ones corresponds to the upper limit $\left(V_{H}\right)$. It is, therefore, evident that complementing a bitstream is equivalent to reversing the input range. Moreover, if the input range is symmetrical $\left[V_{L}, V_{H}\right]=\left[-V_{\text {max }}, V_{\text {max }}\right]$, the bitstream complement is equivalent to the multiplication of the input signal by -1 .

In conventional digital circuits, the addition of two bits produces a 2-bit word, which means an increase in complexity. Another simple solution is to perform addition through interleaving, which merges two bitstreams by duplicating the oversampling frequency. However, it is possible to re-shape a multi-bit word, thus obtaining a bitstream at the same sampling rate [3]. This operation can be done with the circuit shown in Fig. 2: a full adder performs the addition of the two bitstreams, and the output bitstream is taken from the carry-out. The output bitstream $B S_{\text {out }}$ corresponds to $V_{\text {out }}=\left(V_{a}+V_{b}\right) / 2$. The same circuit can be used to obtain the difference between two signals, simply by inverting $V_{b}$.

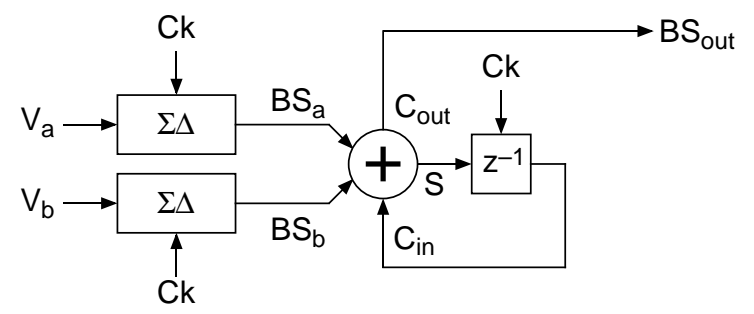

Fig. 2 - Addition of two bitstreams

Multiplication of bitstreams is also straightforward [4]. Indeed, the product of two single-bit signals can be obtained with just one logical gate: an 'AND' if the input range is $\left[0, V_{\text {max }}\right]$ or an 'XNOR' if the input range is $\left[-V_{\max }, V_{\text {max }}\right]$,. However, this approach affects the noiseshaping characteristics. Indeed, multiplication in time domain corresponds to convolution in z-domain. Therefore, the resulting bitstream has four components: one from the convolution of the two signals $S_{1}(z) * S_{2}(z)$, two from convolutions between one signal and the shaped noise of the other bitstream $S_{1}(z) * N_{2}(z)+N_{1}(z) * S_{2}(z)$, and the last from the convolution of the two shaped noises $N_{1}(z) * N_{2}(z)$. Since the last term has a flat frequency spectrum, the result of a multiplication of two noiseshaped bitstreams is a non noise-shaped waveform, whose in-band noise limits the processing accuracy [5]. 
To avoid this drawback, a different multiplication scheme can be used. The input signal $V_{b}$ is converted into a bitstream, which is multiplied by $V_{a}$. Then the resulting signal $V_{\text {out }}$ can be passed through another sigma-delta modulator to obtain the output bitstream $B S_{O u t}$, as shown in Fig. 3 When the signal $V_{b}$ has a single polarity range $\left[0, V_{\text {max }}\right]$, the multiplication block in Fig. 3 can be implemented with an 'on-off' switch. If $V_{b}$ has a symmetrical range $\left[-V_{\max }, V_{\max }\right]$, then the product is made by switching between $V_{a}$ and $-V_{a}$.

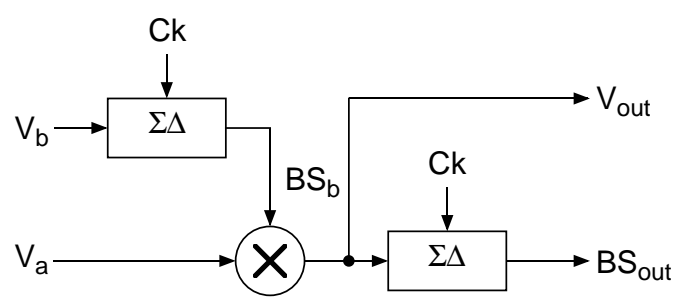

Fig. 3 - Bitstream multiplication of two signals

\section{4. $R M S$ Calculation Unit Implementation}

The block diagram of the proposed digital $R M S$ calculation block is shown in Fig. 4. The circuit exploits the oversampling techniques (bitstreams) described in the previous section to eliminate the area inefficient multipliers, while achieving true $R M S$ calculation.

According to Eqn. (1), the proposed $R M S$ calculation unit includes a squarer, an accumulator and a square root calculator. Since the sign bit can be neglected in the $R M S$ calculation, the input signal of the circuit consists of the 12 least significant bits of the 13 bit A/D converter output word (Fig. 1). Assuming a sampling frequency of $20 \mathrm{kHz}$ the proposed circuit delivers the $R M S$ value of the input signal (maximum frequency $150 \mathrm{~Hz}$ ) within $500 \mathrm{~ms}$.

The input signal $x$ is squared by modulating $x$ with an oversampled version of $x$ (Squarer), according to the bitstream multiplication principle illustrated in Fig. 3. The same squarer block is also used to implement the squareroot calculation, by exploiting a feedback loop with suitable gain (Clipping-Accumulator). The Clipping-Accumulator block, together with the Decimating-Filter blocks, also performs the low-pass filtering function required by the $R M S$ calculation algorithm. Indeed, the feedback loop forces $z^{2}$ (DC signal, in view of the filtering) to equal the average value of $x^{2}$. The resulting output signal $z$, therefore, represents the $R M S$ value of the input signal $x$.

Low-pass decimating filters (Decimating-Filter) are used at the output of both squarer blocks to reconstruct a 12-bit word from the bitstream and filter part of the undesired AC components of the signals. The implementation and the DC gain of the Clipping-Accumulator block were optimized with behavioral simulations to guarantee the stability of the feedback loop under any operating conditions. In particular, we included a clipping function which limits the excursion of the signals. Indeed, the Clipping-Accumulator block accumulates only the sign of $x^{2}-y$ (rounding to \pm 1 ).

With this architecture, using only adders and registers (without multipliers), we drastically reduced (almost by $30 \%$ ) the amount of silicon area required to implement the $R M S$ calculation algorithm for a 13 bit word.

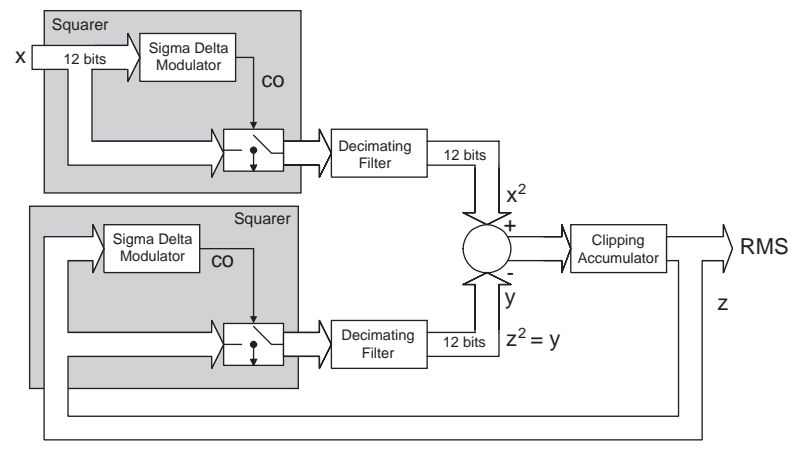

Fig. 4 - Block diagram of the digital RMS calculation unit

\section{Simulation Results}

The proposed RMS calculation unit was designed, simulated and optimized with SIMULINK, implemented in VHDL and then synthesized.

The transient behavior of the system obtained by SIMULINK simulations is illustrated in Fig. 5, Fig. 6, Fig. 7 and Fig. 8. The input signal (Fig. 5) is a rectified sinusoid with amplitude equal to 0.6 (the amplitude of the full scale signal is equal to 1 ). The decimated output signals of the two Squarer blocks, (Fig. 6 and Fig. 7, respectively), after the initial transient, are forced to be equal by the feedback loop, thus producing the correct $R M S$ signal at the system output (Fig. 8).

The system, implemented in VHDL, was synthesized and verified. Fig. 9 shows the signal obtained in the gate-level simulation at the output of the $R M S$ calculation block with a full scale $50 \mathrm{~Hz}$ sinusoidal signal at the input.

\section{Conclusions}

In this paper we proposed a novel 13-bit digital $R M S$ calculation unit, designed to be integrated together with sensor and interface circuits in complete microsystems. The circuit, based on oversampling techniques (bitstream processing), does not require the area inefficient multipliers essential for a traditional $R M S$ calculation unit, thus allowing a significant reduction (almost 30\%) in silicon area. A prototype of the proposed $R M S$ calculation was included in a fully integrated CMOS magnetic current monitor [6]. 


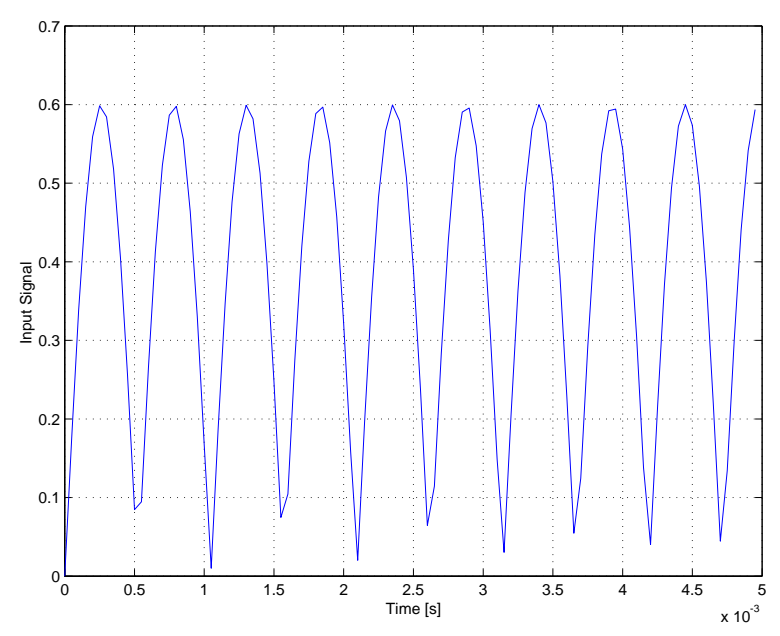

Fig. 5 - Input signal of the proposed RMS calculation unit (50 Hz sinusoidal signal with amplitude 0.6 FS)

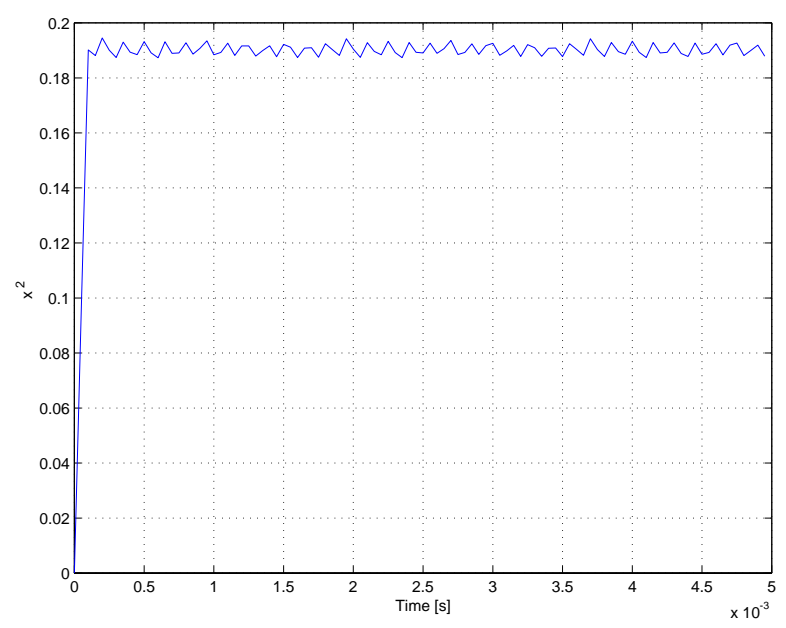

Fig. 6 - Decimated output signal of the squarer calculation block $\left(x^{2}\right)$ with the input signal of Fig. 5

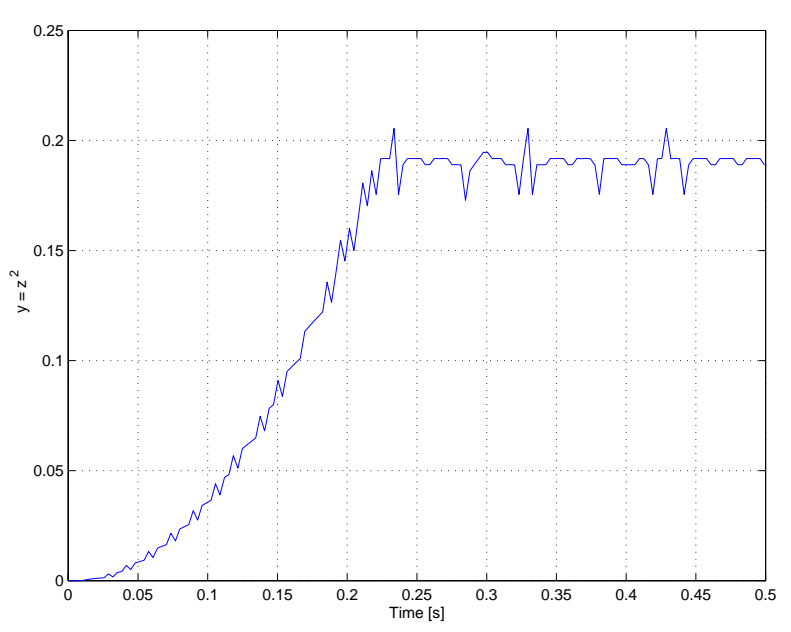

Fig. 7 -Decimated output signal of the square root calculation block $\left(y=z^{2}\right)$ with the input signal of Fig. 5

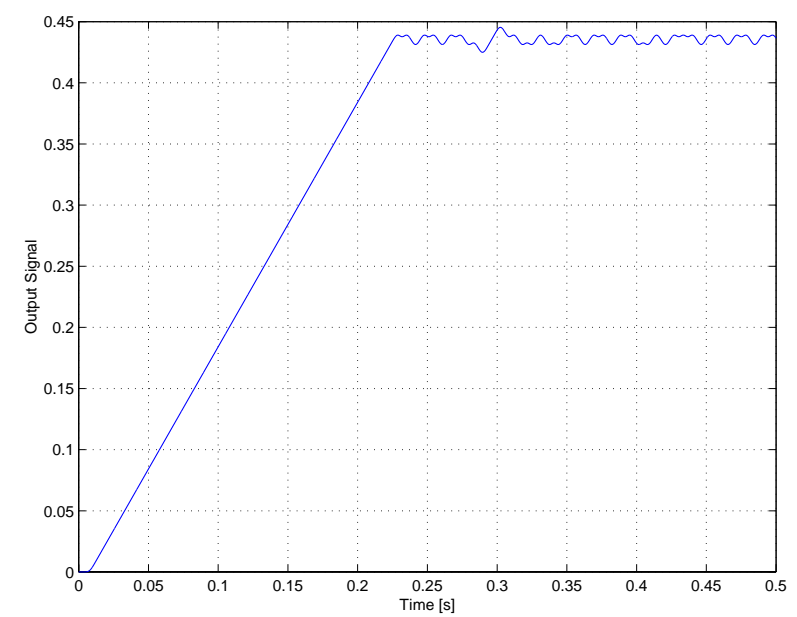

Fig. 8-Simulated output signal of the system with the input signal of Fig. 5

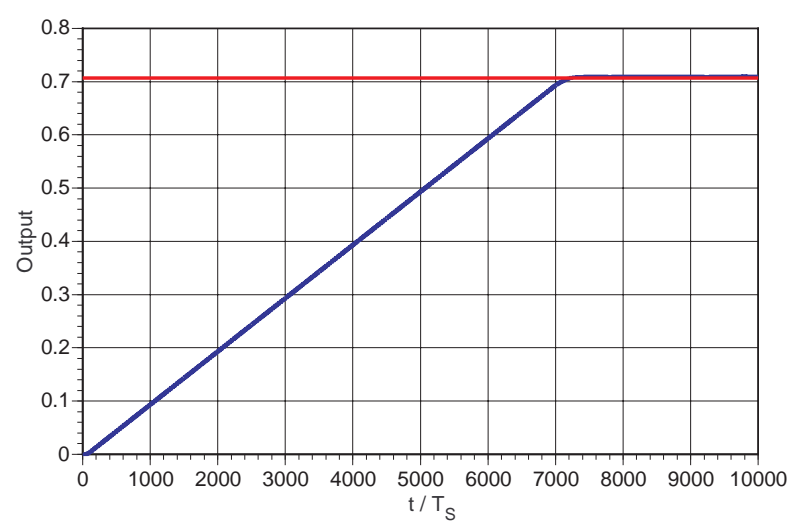

Fig. 9 - Simulated output signal of the RMS calculation unit with a $50 \mathrm{~Hz}$ full scale sinusoidal input

\section{References}

[1] C. F. Coombs, Electronic instrument handbook, McGraw-Hill, New York, NY, 1995.

[2] R. Chapel, "RMS voltage measurements - which method works best?," Electronic Products, 15, pp. 35 (1973).

[3] P. O'Leary and F. Maloberti, "A bitstream adder for oversampling coded data," Electronics Letters, 26, pp. 1708 (1990).

[4] F. Maloberti, "Non conventional signal processing by the use of sigma delta technique: a tutorial introduction," Proc. IEEE Int. Symp. Circ. and Syst., San Diego, CA, 6, 2645 (1992).

[5] V. F. Dias, "Signal processing in the sigma-delta domain,” Microelectronics Journal, 26, 543 (1995).

[6] P. Malcovati and F. Maloberti, "A fully integrated CMOS magnetic current monitor," Proc. IEEE Int. Symp. Circ. and Syst., Orlando, FL, 5, pp. 128 (1999). 